\title{
ON POWER COMPACT OPERATORS
}

\author{
JOSÉ BARRÍA
}

\begin{abstract}
We give an operator theoretic proof of the following result of D. G. Tacon:

THEOREM. If $\left\{T_{n}\right\}$ is a sequence of bounded linear operators in a complex infinite dimensional Hilbert space with the property that for every bounded sequence $\left\{x_{n}\right\}$ there exists a positive integer $k$ such that the sequence $\left\{T_{k} x_{n}\right\}_{n=1}^{\infty}$ has a convergent subsequence, then there exists $k$ such that $T_{k}$ is a compact operator.
\end{abstract}

Let $\mathcal{H}$ be a complex infinite dimensional Hilbert space, and let $\mathcal{L}(\mathcal{H})$ denote the algebra of all bounded linear operators on $\mathcal{H}$. We shall say that a sequence $\left\{T_{n}\right\}$ in $\mathcal{L}(\mathcal{H})$ has property (P) if for every bounded sequence $\left\{x_{n}\right\}$ in $\mathcal{H}$ there exists a positive integer $k$ such that $\left\{T_{k} x_{n}\right\}_{n=1}^{\infty}$ has a convergent subsequence. In [1] D. G. Tacon, using techniques from nonstandard analysis, showed that every sequence with property $(\mathrm{P})$ contains a compact operator. More generally, in [1] this result is proved for sequences of operators acting on a Banach space. The purpose of this paper is to give a proof of this theorem in the standard framework of operator theory for the case when the operators are acting in a complex Hilbert space. Our proof is based on a deep theorem of D. Voiculescu [2]. The result we need from [2] is stated as Theorem A below. First we prove two elementary lemmas.

LEMMA 1. Let $\left\{T_{n}\right\}$ be a sequence in $\mathcal{L}\left(\mathcal{H}_{1}\right)$ with property (P). If $U: \mathcal{K}_{1} \rightarrow \mathcal{H}_{2}$ is a unitary operator and $\left\{K_{n}\right\}$ is a sequence of compact operators in $\mathcal{L}\left(\mathcal{H}_{2}\right)$, then the sequence $\left\{U T_{n} U^{*}+K_{n}\right\}$ has property $(\mathrm{P})$.

Proof. Property (P) is preserved by compact perturbations of the operators in the sequence, and obviously the sequence $\left\{U T_{n} U^{*}\right\}$ has property $(\mathrm{P})$.

Lemma 2. Let $A_{n} \in \mathcal{L}(\mathcal{H})$ such that $A_{n} \neq 0$ for $n=1,2, \ldots$ Then there exists $x_{0}$ in $\mathcal{H}$ such that $A_{n} x_{0} \neq 0$ for all $n$.

Proof. It is an easy consequence of the Baire category theorem.

We denote the ideal of compact operators in $\mathcal{L}(\mathcal{H})$ by $K(\mathcal{H})$, and the quotient map of $\mathcal{L}(\mathcal{K})$ onto the Calkin algebra $\mathcal{L}(\mathcal{H}) / K(\mathcal{H})$ by $\pi$. Let $\mathcal{K}_{\infty}=\mathscr{K} \oplus \mathscr{K}$ $\oplus \ldots$ be the direct sum of $\aleph_{0}$ copies of the Hilbert space $\mathcal{K}$.

Received by the editors July 9, 1979.

AMS (MOS) subject classifications (1970). Primary 47B05.

Key words and phrases. Compact operator. 
THEOREM A [2]. Let $T_{n} \in \mathcal{L}(\mathcal{H C})(n=1,2, \ldots)$ and let $\mathbb{Q}$ be the separable $C^{*}$-subalgebra of $\mathcal{L}(\mathcal{F C}) / K(\mathcal{F C})$ generated by $\pi(I)$ and the sequence $\left\{\pi\left(T_{n}\right)\right\}$. Let $\rho$ be a faithful representation of $\mathcal{Q}$ on some separable Hilbert space $\mathcal{K}$. Then there exists a unitary operator $U: \mathcal{H} \rightarrow \mathcal{H} \oplus \mathcal{K}_{\infty}$ such that $U T_{n} U^{*}-T_{n} \oplus \rho\left(\pi\left(T_{n}\right)\right) \oplus \rho\left(\pi\left(T_{n}\right)\right)$ $\oplus \ldots$ is a compact operator for $n=1,2, \ldots$

THEOREM B [1]. Let $\left\{T_{n}\right\}$ be a sequence in $\mathcal{L}(\mathcal{H C})$ with property $(\mathrm{P})$. Then there exists a positive integer $k$ such that $T_{k}$ is a compact operator.

Proof. Suppose the conclusion of the theorem were false. Then $\pi\left(T_{n}\right) \neq 0$ for $n=1,2, \ldots$ If we apply Theorem A to the sequence $\left\{T_{n}\right\}$, then $K_{n}+U T_{n} U^{*}=$ $T_{n} \oplus A_{n} \oplus A_{n} \oplus \ldots$ where $K_{n}$ is a compact operator on $\mathcal{H} \oplus \mathcal{K}_{\infty}$ and $A_{n}=$ $\rho\left(\pi\left(T_{n}\right)\right) \in \mathcal{L}(\mathcal{K})$. From Lemma 1 the sequence defined by $T_{n}^{\prime}=T_{n} \oplus A_{n} \oplus A_{n}$ $\oplus \ldots$ has property (P). Since $\rho$ is a faithful representation, we have that $A_{n} \neq 0$ for all $n$. Then from Lemma 2 there exists a vector $y$ in $\mathcal{K}$ such that $A_{n} y \neq 0$ for all $n$.

Let $\left\{x_{n}\right\}$ be the bounded sequence in $\mathcal{H} \oplus \mathcal{K}_{\infty}$ defined by

$$
x_{n}=\left\langle\widetilde{0, \ldots, 0}_{n}, 0,0, \ldots\right\rangle \quad(n=1,2, \ldots) .
$$

Then

$$
T_{k}^{\prime} x_{n}=\overbrace{\langle 0, \ldots, 0}^{n}, A_{k} y, 0,0, \ldots\rangle
$$

and $\left\|T_{k}^{\prime} x_{n}-T_{k}^{\prime} x_{m}\right\|=\sqrt{2}\left\|A_{k} y\right\|$ for $n \neq m$ and $k=1,2, \ldots$ This shows that the sequence $\left\{T_{n}^{\prime}\right\}$ does not have property $(\mathrm{P})$. This is a contradiction. Therefore $\pi\left(T_{k}\right)=0$ for some positive integer $k$ and $T_{k}$ is a compact operator.

REMARK [1]. If we consider a sequence of the form $\left\{T^{n}\right\}$ with a fixed operator $T$, then property $(\mathrm{P})$ is a necessary and sufficient condition for the operator $T$ to have a compact power.

\section{REFERENCES}

1. D. G. Tacon, Two characterizations of power compact operators, Proc. Amer. Math. Soc. 73 (1979), 356-360.

2. D. Voiculescu, A noncommutative Weyl-von Neumann theorem, Rev. Roumaine Math. Pures Appl. 21 (1976), 97-113.

Departamento de Matemáticas, Instituto Venezolano de Investigaciones Científicas, A. P. 1827, Caracas 101, Venezuela 